Meta

Journal des traducteurs

Translators' Journal

\title{
La Stylistique comparée du français et de l'anglais : la théorie au service de la pratique
}

\section{Isabelle Collombat}

Volume 48, numéro 3, septembre 2003

Traduction et enseignement

Translation and teaching

URI : https://id.erudit.org/iderudit/007602ar

DOI : https://doi.org/10.7202/007602ar

Aller au sommaire du numéro

Éditeur(s)

Les Presses de l'Université de Montréal

ISSN

0026-0452 (imprimé)

1492-1421 (numérique)

Découvrir la revue

Citer cet article

Collombat, I. (2003). La Stylistique comparée du français et de l'anglais : la théorie au service de la pratique. Meta, 48(3), 421-428.

https://doi.org/10.7202/007602ar
Résumé de l'article

L'enseignement de la traduction au premier cycle vise à faire acquérir aux apprenants un savoir-faire professionnel ; dans cette optique, la dialectique entre théorie et pratique y revêt une importance particulière. C'est d'ailleurs la confusion entre les deux types de théorie - que nous nommerons respectivement " instrumentale " et " spéculative » - qui a été à l’origine des critiques les plus virulentes contre la Stylistique comparée du français et de l'anglais (SCFA) de Vinay et Darbelnet. Le présent article, fondé sur un sondage mené auprès d'étudiants de première année en traduction, a pour objet de montrer que la SCFA ressortit à la théorie instrumentale et qu'en ce sens, elle ne s'oppose pas à la pratique mais interagit avec elle. 


\title{
La Stylistique comparée du français et de l'anglais: la théorie au service de la pratique
}

\author{
ISABELLE COLLOMBAT \\ Université Laval, Québec, Canada \\ icollombat@videotron.ca
}

\begin{abstract}
RÉSUMÉ
L'enseignement de la traduction au premier cycle vise à faire acquérir aux apprenants un savoir-faire professionnel; dans cette optique, la dialectique entre théorie et pratique y revêt une importance particulière. C'est d'ailleurs la confusion entre les deux types de théorie - que nous nommerons respectivement «instrumentale » et «spéculative » - qui a été à l'origine des critiques les plus virulentes contre la Stylistique comparée du français et de l'anglais (SCFA) de Vinay et Darbelnet. Le présent article, fondé sur un sondage mené auprès d'étudiants de première année en traduction, a pour objet de montrer que la SCFA ressortit à la théorie instrumentale et qu'en ce sens, elle ne s'oppose pas à la pratique mais interagit avec elle.
\end{abstract}

\section{ABSTRACT}

As undergraduate translation training is designed to help learners acquire professional competence, the dialectics between theory and practice takes on special importance. The confusion between two types of theory - which we refer to as "instrumental" and "speculative" respectively - has engendered the most virulent criticism of Vinay and Darbelnet's Comparative Stylistics of French and English (CSFE). Based on a survey of first year translation students, this paper aims to show that CSFE pertains to instrumental theory and is therefore not incompatible with it, but rather interacts with it.

\section{MOTS-CLÉS/KEYWORDS}

stylistique comparée, théorie, pratique, pédagogie de la traduction

La théorie n'est que l'idée scientifique contrôlée par l'expérience.

Claude Bernard (1954: 45)

En 1957, Jean-Paul Vinay annonçait la naissance d'une «nouvelle science, la stylistique comparée» (1957: 141-148). L’année suivante, il publiait avec Jean Darbelnet la première édition de la Stylistique comparée du français et de l'anglais (SCFA). Si la SCFA «repose sur des principes théoriques", elle n'en est pas "pour autant [...] une théorie de la traduction» (Vinay 1983: 425). En se fondant sur des concepts théoriques linguistiques, Vinay et Darbelnet créent de nouvelles notions - comme en témoigne le grand nombre de néologismes de la SCFA - permettant de formaliser, de modéliser la pratique à des fins pédagogiques. Leur postulat est le suivant: «Les démarches du traducteur et du stylisticien comparatif sont intimement liées, bien que de sens contraire. La stylistique comparée part de la traduction pour dégager ses lois; le traducteur utilise les lois de la stylistique comparée pour bâtir sa traduction » (1958: 21). Ainsi, dès les prémisses, les «inventeurs» de la stylistique comparée revendiquaient une place originale, entre théorie et pratique, les deux agissant en interaction.

Meta, XLVIII, 3, 2003 
Nous allons tout d'abord nous attacher à définir la ou les théorie(s) dont il est ici question, puis exposer brièvement l'intérêt présenté par la SCFA dans l'apprentissage de la traduction. Ensuite, nous présenterons les résultats d'un petit sondage mené auprès d'étudiants du cours de grammaire et stylistique différentielles et les quelques conclusions que l'on peut en tirer.

\section{Quelle théorie?}

Avant d'examiner l'interaction entre théorie et pratique dans la méthode de traduction proposée par Vinay et Darbelnet, il paraît judicieux de s'interroger sur le terme même de théorie. La définition de la pratique («Activités volontaires visant des résultats concrets [opposé à théorie] $\left.{ }^{1} »\right)$ ne semble pas quant à elle poser de difficulté particulière ni être source d'ambiguïté.

La diversité des définitions proposées de la théorie est manifeste dans l'article correspondant du Nouveau Petit Robert, où l'on trouve les définitions suivantes:

1. Ensemble d'idées, de concepts abstraits, plus ou moins organisés, appliqué à un domaine particulier. Bâtir une théorie. Théories artistiques. Les théories politiques. [...] Mettre une théorie en application.

2. sc Construction intellectuelle méthodique et organisée, de caractère hypothétique (au moins en certaines de ses parties) et synthétique. Principes et lois d'une théorie. Théorie des ensembles. Théorie de la relativité.

Éléments de connaissance organisés en système (dans un but didactique). La théorie musicale.

De l'examen de ces définitions, il ressort qu'il existe deux types principaux de théorie, que nous nommerons respectivement théorie «instrumentale» ou «normative» (par exemple, grammaire, stylistique, solfège) et théorie «spéculative» (par exemple, herméneutique, musicologie), la première encadrant selon nous la «transmission d'un savoir-faire», pour reprendre les mots de Durieux (1988:115) et la seconde, la modélisation d'un savoir.

Cette distinction entre les deux formes de théorie est fondamentale, car c'est la confusion entre théorie instrumentale et théorie spéculative qui a déterminé les critiques les plus virulentes à l'égard de la SCFA. Pour Roberts (1984: 49), en effet, les détracteurs qui reprochaient à la méthode de Vinay et Darbelnet de ne pas « [présenter] une théorie de la traduction, mais un ensemble de recettes pour traducteurs » fondaient leur critique sur «un malentendu sur le sens du mot "théorie" ».

\section{Théorie et pratique en traduction}

En traduction, la dialectique entre théorie et pratique revêt une importance particulière; on pourrait même avancer qu'il s'agit là d'un sujet sensible, si l'on en juge par la manière dont les pédagogues de la traduction se défendent tantôt d'être trop théoriques, tantôt d'être trop pratiques, comme la lecture des préfaces et avant-propos des méthodes de traduction nous l'enseigne: «La théorie n'est cependant jamais dissociée de la pratique», écrit Jacqueline Guillemin-Flescher en préface de l'ouvrage d'Hélène Chuquet et Michel Paillard, Approche linguistique des problèmes de traduction anglais - français. "Ceci n'est ni une grammaire ni un ouvrage de linguistique mais un essai d'initiation à et de réflexion sur la traduction. [...] Rien de ce qui est avancé 
ici n'est le fait d'une théorisation abstraite et ne fonctionnant que pour elle-même », écrit à son tour Michel Ballard (1994) en avant-propos. "Plutôt que de longues dissertations théoriques abstraites, l'auteur a eu le courage scientifique de n'écrire que pour ses utilisateurs ", affirme Georges Mounin dans sa préface de Claude Tatilon (1986).

La traduction est généralement considérée comme une matière orientée vers la pratique professionnelle et partant, l'enseignement de la traduction vise à former des professionnels de la traduction armés d'un savoir-faire. En ce sens, en traduction, la théorie telle qu'on l'oppose à la pratique est de nature instrumentale, et non spéculative. Elle le devient aux cycles supérieurs, où l'on traite non plus spécifiquement de traduction, mais de traductologie.

Quoi qu'il en soit, les formateurs reconnaissent que ces zones de tangence entre théorie et pratique sont généralement facteurs de progrès, autant pour l'apprentitraducteur que pour le traducteur formé: le premier recourt à la théorie instrumentale, lorsqu'il est en phase d'acquisition des outils «susceptibles de le guider dans les choix à faire lorsqu'il traduit» (Valentine 1998: 13) et le second se tourne parfois vers la théorie spéculative, quand il «réfléchit sur sa pratique», car «la traductologie est nécessaire pour bien comprendre l'opération traduisante, et mieux traduire» (Flamand 1983: 40-41).

\section{Pourquoi la Stylistique comparée du français et de l'anglais?}

Nous avons choisi de fonder la présente étude sur la Stylistique comparée du français et de l'anglais de Vinay et Darbelnet pour plusieurs raisons concomitantes: le cours de grammaire et de stylistique différentielles donné aux étudiants de première année en traduction à l'Université Laval est fondé en grande partie sur la méthode de Vinay et Darbelnet. Il existe certes de nombreux autres manuels de traduction, mais ils reprennent très souvent la méthode de Vinay et Darbelnet, en particulier en ce qui a trait aux sept procédés de traduction qu'ils ont dégagés. La SCFA est généralement considérée comme «la première méthode de traduction fondée sur une analyse scientifique» et élaborée «dans un but pédagogique» (Oseki-Dépré, 1999: 56). Il nous semble donc judicieux de revenir aux sources.

Par ailleurs, le flot de critiques (positives et négatives) qui a accueilli la parution de la Stylistique comparée du français et de l'anglais en 1958 - et qui ne s'est pas vraiment tari depuis - ne peut que nous conduire à nous interroger et à examiner plus avant cette méthode de traduction. Vinay (1983: 418) signalait à ce titre en 1983, année des 25 ans de la méthode: «[N]ous avons recueilli un large éventail de commentaires, allant du pire au meilleur.» Et il cite: «élémentaire; un tantinet raciste; empirique et parfois confus; produit d'une pratique traductrice totalement incontrôlée; remarquable; admirable; fondamental; vaut bien cinq étoiles; est plein de vues pénétrantes; se lit comme un roman; marque un grand tournant dans l'histoire de la traductologie; bref, l'un des meilleurs manuels de traduction qui soient».

Ces contradictions ne sont pas les seules que l'on peut relever dans l'exégèse de la SCFA. En effet, en pédagogie de la traduction, on oppose généralement deux méthodes: la méthode comparative ou contrastive (celle de Vinay et Darbelnet) qui, selon ses détracteurs, est «trop axée sur la "langue" ( (Roberts 1984: 48), et la méthode interprétative, formalisée notamment par Seleskovitch et Lederer (1984). 
Cette dernière approche consiste en une théorie du sens, et repose sur le triptyque «compréhension - déverbalisation - réexpression». Or, la méthode de Vinay et Darbelnet comprend elle aussi une théorie du sens: "Il y a des cas [...] où la traduction ne ressort ni de la structure, ni du contexte, mais où le sens global et ultime n'est perceptible que pour celui qui connaît la situation.» (1958: 173). Et plus loin: «Les situations ne se trouvent pas dans les dictionnaires» (1958:174) ou encore: «L'étude des situations est $[\ldots]$ essentielle en stylistique comparée, puisqu'elle seule permet de décider, en dernier ressort, de la signification d'un message» (1958: 175). Nous ne prétendons pas ici aller plus avant dans la comparaison des deux approches; Roberts (1984: 48-81) a produit en 1984 une analyse comparative détaillée qui, selon nous, demeure une référence incontournable.

Pour conclure cet exposé succinct des raisons de notre choix, nous reprendrons les propos de Rochard (2000): «Traduire, c'est un dialogue permanent entre le contrastif et l'interprétatif». Il nous semble à ce titre que la méthode de Vinay et Darbelnet autorise précisément ce va-et-vient entre sens et structure, ce syncrétisme entre les deux «doctrines» que l'on oppose généralement. C'est pourquoi elle constitue selon nous une base pédagogique pertinente.

\section{L'avis des étudiants}

Puisqu'il s'agit de réfléchir à la pédagogie de la traduction, il nous a paru opportun de consulter les destinataires de l'enseignement, soit les étudiants. Nous avons donc rédigé un questionnaire adressé par courriel à 69 étudiants de grammaire et stylistique différentielles. Les questions posées étaient les suivantes:

1. Selon vous, quelle est l'utilité de la théorie en traduction?

2. À votre avis, l'approche de la traduction proposée par Vinay et Darbelnet dans la Stylistique comparée du français et de l'anglais représente-t-elle un juste équilibre entre théorie et pratique?

oui non (effacez la réponse ne correspondant pas à votre choix)

Expliquez pourquoi:

3. L'apprentissage de la méthode proposée par Vinay et Darbelnet vous a-t-elle selon vous permis d'améliorer votre façon de traduire?

oui non (effacez la réponse ne correspondant pas à votre choix)

Expliquez de quelle manière:

4. D'après vous, peut-on dissocier la théorie et la pratique dans le processus d'apprentissage de la traduction?

oui non (effacez la réponse ne correspondant pas à votre choix)

Expliquez pourquoi:

Commentaires (facultatif):

La réponse à ce questionnaire n'était ni obligatoire ni sanctionnée par une note; on peut donc considérer que, même si le taux de réponse est relativement faible $(20,30 \%$, soit 14 réponses sur 69$)$, il n'en témoigne pas moins d'un certain intérêt de 
la part des étudiants. Certes, la portée des résultats est minorée par ce faible taux de retour; toutefois, ce petit exercice permet de dégager des tendances, comme le montre la synthèse qui suit.

\section{Selon vous, quelle est l'utilité de la théorie en traduction?}

D’une manière générale, les réponses des étudiants ont été influencées par le cours qu'ils venaient de suivre, y compris dans l'emploi de la terminologie; dans leur grande majorité, ils affirment que la théorie permet de mieux différencier les deux langues en présence dans le processus de traduction et de produire en français des traductions idiomatiques et grammaticalement correctes. «La théorie nous aide [...] à éviter les anglicismes, les calques, les barbarismes, à écrire de façon idiomatique.»

Grâce à la théorie, «le traducteur est apte à reconnaitre plus facilement les difficultés linguistiques et les différences qui existent entre la langue de départ et la langue d'arrivée.»

«La théorie nous dicte des solutions à envisager en pratique.»

L'un des répondants - qui se démarque de l'ensemble - aborde la question à contre-courant. Il affirme en préambule: «Je travaille déjà dans le domaine alors je sais de quoi je parle.» Selon cet étudiant, la théorie n'est d'aucune utilité: «La révision que l'on fait après avoir traduit un texte se résume à vérifier les fautes d'orthographe, de sens et de stylistique par exemple et non pas à essayer de trouver les procédés que l'on a utilisés (je ne regarde vraiment pas ça lorsque je traduis).» Il n'empêche qu'il semble paradoxalement appliquer des critères de contrôle de la qualité issus de théories instrumentales (grammaire, notamment). De fait, on pourrait se demander si le fait d'avoir commencé à exercer avant d'étudier n'a pas conduit l'étudiant en question à prendre la théorie instrumentale pour une théorie spéculative, dans la mesure où sa pratique préexistait et où il n'a pas considéré la théorie instrumentale comme constitutive de sa formation professionnelle, mais plutôt comme une réflexion a posteriori sur sa pratique. A contrario, un autre répondant qui signale lui aussi avoir "déjà fait de la traduction avant le bac» affirme que «la théorie nous permet d'être conscients de certains pièges et de certaines difficultés qu'on n'aurait peut-être pas pu identifier par nos propres moyens.»

Selon deux répondants, l'apprentissage de la théorie (en particulier, des procédés de traduction) permet de combler le manque d'expérience: «La théorie permet selon moi d'acquérir des réflexes de traduction qui, autrement, viendraient peut-être avec l'expérience, mais ce n'est pas certain.»

Enfin, pour trois répondants, la théorie permet de traduire "consciemment», car elle permet au traducteur d'«identifier, de classer et de "concrétiser" les processus intellectuels qui s'opèrent en traduisant.»

2. À votre avis, l'approche de la traduction proposée par Vinay et Darbelnet dans la Stylistique comparée du français et de l'anglais représente-t-elle un juste équilibre entre théorie et pratique?

À cette question précise, $71,43 \%$ des répondants (10 sur 14) ont répondu par l'affirmative, soulignant que les aspects théoriques évoqués par Vinay et Darbelnet étaient toujours accompagnés de "nombreux exemples pertinents" "dans lesquels la théorie est mise en pratique». À noter cette remarque d'un répondant: "Les exemples remplacent un peu la pratique... ». L'un des répondants affirme même: «On tentait de prouver 
que les bases démontrées étaient bel et bien des bases appliquées et non une théorie sans réel fondement. " Un autre a formulé une remarque intéressante, qui pourrait à elle seule infirmer certaines critiques selon lesquelles la SCFA serait par trop «lexicaliste»: "Comparer le système linguistique des deux langues permet de comprendre que traduire n'est pas seulement rendre les mots de la langue de départ par des mots équivalents dans la langue d'arrivée. En fait, c'est passer d'un système à un autre. La théorie présentée facilite ce passage.»

3. L'apprentissage de la méthode proposée par Vinay et Darbelnet vous a-t-elle selon vous permis d'améliorer votre façon de traduire?

À cette question, $92,85 \%$ des répondants (13 sur 14) ont répondu par l'affirmative. D’une manière générale, ils se disent rassurés (trois étudiants le signalent) de pouvoir nommer les difficultés de traduction abordées et d'être aptes à les repérer et à les résoudre de manière consciente.

Deux étudiants ont répondu «non" à la question précédente, mais «oui » à celle-ci: s'ils considèrent que la SCFA manque d'applications pratiques, ils expliquent néanmoins qu'elle les a accoutumés à recourir à un certain raisonnement avant de traduire et à éviter consciemment les écueils de la traduction de l'anglais au français. Selon ces deux répondants, la méthode de Vinay et Darbelnet ne représente pas un juste équilibre entre théorie et pratique, car elle n'intègre pas d'exercices permettant de mettre en pratique les notions théoriques; or, la SCFA s'accompagne bien de deux cahiers d'exercices, que nous n'avons pas utilisés dans le cours, préférant faire travailler les étudiants sur un cahier d'exercice de notre cru, plus actuel.

4. D'après vous, peut-on dissocier la théorie et la pratique dans le processus d'apprentissage de la traduction?

À cette question, 92,85\% des répondants (13 sur 14) ont répondu par la négative. De manière plus ou moins consciente, les étudiants ont mis le doigt sur l'inextricable interaction entre théorie et pratique, lesquelles s'alimentent mutuellement: "On ne peut pas apprendre qu'en écoutant ou en lisant. Il faut se pratiquer. De même, on ne peut pas apprendre qu'en pratiquant.» Autre remarque intéressante: "D'après moi, [la théorie et la pratique] se complètent pour former des traducteurs compétents qui savent faire les choses, comment les faire et pourquoi ils les font.»

De l'ensemble des réponses aux questions, il ressort que les étudiants attendent de la théorie qu'elle les aide à prendre conscience de leur manière de traduire, en leur fournissant le cas échéant des outils d'autoévaluation: «J'aime mettre en pratique les éléments de théorie que j'ai appris, ce qui permet aussi une forme d'évaluation personnelle.» De même, les étudiants s'avèrent sensibles à la validation de la théorie par la pratique et réciproquement; ils ont besoin d'être convaincus. C'est également ce qui ressortait du constat de Durieux, lorsqu'elle affirme: «Les rappels théoriques par le responsable d'un cours de traduction technique seront fréquents pour convaincre son auditoire du bien-fondé de la méthode et de son efficacité pratique» (1988: 118), ou encore: «L'exposé de principes théoriques ne convaincra pas les étudiants s'ils ne se trouvent eux-mêmes placés dans des situations concrètes» (1988: 112).

Autre constat intéressant: les étudiants interrogés semblent considérer la théorie instrumentale comme un concentré d'expérience: 
«Comme dans tout art, la théorie est un outil nécessaire à l'apprentissage parce qu'elle nous fait comprendre, nous fait voir des choses qui sans elle, nous prendraient beaucoup de temps à comprendre et à réaliser. [...] Je crois que la théorie est un “accélérateur"dans notre apprentissage.» «Lorsqu'on passe à la pratique, la théorie permet d'améliorer la rapidité d'exécution du traducteur et la qualité de son travail.»

La SCFA est-elle toujours d'actualité?

«On prétend [...] que [la SCFA] est "démodée", "dépassée", que certaines "théories" modernes expliquent mieux la traduction", affirmait Roberts en 1984. C'était il y a presque 20 ans, et la question reste... d'actualité. Parmi les répondants à notre sondage, l'un a signalé qu'il serait plus adapté de travailler avec des ouvrages écrits après 1990 et un autre a mentionné: "L'ouvrage date un peu et à cause de cela certaines choses s'appliquent moins.» Certains autres ont évoqué la présentation matérielle de l'ouvrage - jugée peu attrayante - et le style classique, qu'ils avaient parfois du mal à comprendre.

On peut juger ces observations fondées; s'agissant notamment des équivalences de situation ou adaptations, on trouve dans la SCFA des exemples qui peuvent faire sourire aujourd'hui (par exemple, adaptation des métaphores sportives: cricket, baseball, cyclisme), à une époque où tout fossé culturel ressenti comme tel peut être comblé en un clic de souris grâce à Internet. Vinay (1983: 418), lui-même conscient que l'ouvrage pouvait être amélioré, cite Kahn (1970-1972: 21), qui considérait la SCFA comme "un ouvrage de pionniers, qui demande à être refondu sur une base plus rigoureuse et précise». La publication en 1995 de la traduction anglaise de la SCFA a été l'occasion d'une légère actualisation des exemples, mais cette «rénovation» est mineure.

Au-delà de ce constat et pour conclure, nous en reviendrons à la question de la différence entre théorie instrumentale et théorie spéculative: il est rare qu'on entende dire d'une grammaire ou d'un ouvrage de solfège qu'ils se démodent sur le fond, parce qu'ils font état de règles normatives destinées à structurer un savoir-faire et non de spéculations. En revanche, au plan de la traductologie, le débat entre sourciers et ciblistes, par exemple, est daté : l'histoire de la traduction montre qu'il a bel et bien existé des modes en ce domaine. Certes, sur la forme, un toilettage de la SCFA serait le bienvenu (actualisation des exemples, intégration des nouvelles technologies de l'information, etc.), mais dans la mesure où la démarche proposée par Vinay et Darbelnet ressortit à la théorie instrumentale, la SCFA ne saurait dater sur le fond. Sans doute, il incombe au formateur de l'actualiser et de la rendre plus attrayante sur la forme, notamment en l'associant aux nouvelles technologies qui constituent le quotidien du traducteur d'aujourd'hui.

\section{NOTES}

1. Le nouveau Petit Robert, 1993.

\section{RÉFÉRENCES}

Ballard, M. (1994): La traduction de l'anglais au français, $2^{\mathrm{e}}$ édition, Paris, Nathan.

Bernard, C. (1954): Introduction à l'étude de la médecine expérimentale, Paris, Les éditions de

l'École. 
Chuquet, H. et M. Paillard (1987): Approche linguistique des problèmes de traduction anglaisfrançais, Paris, OPHRYS.

Durieux, C. (1988) : Fondement didactique de la traduction technique, Paris, Didier Érudition.

Flamand, J. (1983) : Écrire et traduire: sur la voie de la création, Ottawa, Éditions du Vermillon.

Guével, Z. et E. Valentine, dir. (1998): Traduction et langues de spécialité, Approches théoriques et considérations pédagogiques, Québec, Centre International de recherche en aménagement linguistique, Université Laval.

Kann, F. (1970-1972): «Traduction et linguistique», dans Cahiers Ferdinand de Saussure, n 27. Oseri-Dépré, I. (1999): Théories et pratiques de la traduction littéraire, Paris, Armand Colin.

Le nouveau Petit Robert, Dictionnaire alphabétique et analogique de la langue française (1993), texte remanié et amplifié sous la direction de Josette Rey-Debove et Alain Rey, Paris, Dictionnaires Le Robert.

Roberts, R. P. (1984): «La stylistique comparée du français et de l'anglais en 1980 », dans A. Thomas et J. Flamand (dir.) La traduction: l'universitaire et le praticien, Ottawa, Les Presses de l'Université d'Ottawa.

Rochard, M. (2000) : «L'interprétatif et le contrastif sont-ils solubles dans la logique? », extraits d'une contribution présentée au colloque de l'Association canadienne de traductologie, Edmonton, juin 2000, <http://perso.wanadoo.fr/michel.rochard/Methodologie/Edmonton. html>.

Seleskovitch, D. et M. Lederer (1984): Interpréter pour traduire, Paris, Publications de la Sorbonne, Didier Érudition.

TAtilon, C. (1986): Traduire, pour une pédagogie de la traduction, Toronto, Éditions du GREF.

Vinay, J.-P. et J. Darbelnet (1958): Stylistique comparée du français et de l'anglais, Montréal, Beauchemin.

Vinay, J.-P. et J. Darbelnet (1995): Comparative Stylistics of French and English: a Methodology for Translation, traduit par Juan C. Sager et M.-J. Hamel, Amsterdam, John Benjamins.

VinAY, J.-P. (1957) : «Peut-on enseigner la traduction? ou Naissance de la Stylistique comparée», Meta, 2-4, p. 141-151.

VINAY, J.-P. (1983) : «SCFA Revisited», Meta, 28-4, p. 417-431. 